\title{
Acoustic waves in the solar atmosphere at high spatial resolution
}

\author{
N. Bello González ${ }^{1,2}$, M. Flores Soriano ${ }^{2}$, F. Kneer ${ }^{2}$, and O. Okunev ${ }^{2,3}$ \\ 1 Kiepenheuer-Institut für Sonnenphysik, Schöneckstr. 6, 79104 Freiburg, Germany \\ e-mail: nbello@kis.uni-freiburg.de \\ 2 Institut für Astrophysik, Friedrich-Hund-Platz 1, 37077 Göttingen, Germany \\ e-mail: kneer@astro.physik.uni-goettingen.de; olok@astro.physik.uni-goettingen.de \\ 3 Central Astronomical Observatory of the Russian Academy of Sciences, Pulkovskoye chaussee 65/1, 196140 St. Petersburg, Russia
}

Received 4 April 2009 / Accepted 10 August 2009

\begin{abstract}
Aims. The energy supply for the radiative losses of the quiet solar chromosphere is studied. On the basis of high spatial resolution data, we investigate the amount of energy flux carried by acoustic waves in the solar photosphere.

Methods. Time sequences from quiet Sun disc centre were obtained with the "Göttingen" Fabry-Perot spectrometer at the Vacuum Tower Telescope, Observatorio del Teide/Tenerife, in the non-magnetic Fe I 5576 A line. The data were reconstructed with speckle methods. The velocity and intensity fluctuations at line minimum were subjected to Fourier and wavelet analyses. The energy fluxes at frequencies higher than the acoustic cutoff frequency (period $U \approx 190 \mathrm{~s}$ ) were corrected for the transmission of the solar atmosphere, which reduces the signal from short-period waves.

Results. Both Fourier and wavelet analysis give an amount of energy flux of $\sim 3000 \mathrm{~W} \mathrm{~m}^{-2}$ at a height $h=250 \mathrm{~km}$. Approximately $2 / 3$ of it is carried by waves in the $5-10 \mathrm{mHz}$ range, and $1 / 3$ in the $10-20 \mathrm{mHz}$ band. Extrapolation of the flux spectra gives an energy flux of 230-400 W m $\mathrm{m}^{-2}$ at frequencies $v>20 \mathrm{mHz}$. We find that the waves occur predominantly above inter-granular areas. Conclusions. We conclude that the acoustic flux in waves with periods shorter than the acoustic cutoff period can contribute to the basal heating of the solar chromosphere, in addition to the atmospheric gravity waves found recently.
\end{abstract}

Key words. Sun: photosphere - Sun: chromosphere - Sun: oscillations - techniques: high angular resolution techniques: spectroscopic

\section{Introduction}

According to standard, hydrostatic models of the solar atmosphere (Vernazza et al. 1981; Maltby et al. 1986), the net radiative losses from the quiet solar chromosphere amount to approximately $4600 \mathrm{Wm}^{-2}$ (Vernazza et al. 1981) to $14000 \mathrm{~W} \mathrm{~m}^{-2}$ (Anderson \& Athay 1989). The non-radiative, (magneto-)mechanical supply of this flux represents a longlasting and yet unsolved problem for the physics of the outer solar and stellar atmospheres.

Biermann (1948) and Schwarzschild (1948) suggested acoustic waves, generated by turbulent convection, for the energy transport and heating. This has been pursued since then in numerous theoretical and observational studies. We mention here the work by Ulmschneider and his Heidelberg group (e.g. Fawzy et al. 2002). The reader may trace back from this reference much of earlier work on acoustic (as well as magnetic) heating of chromospheres. Such studies were designed to simulate an averaged chromospheric temperature rise as a result of shock dissipation of short-period wave energy, with periods in the range $U=10 \ldots 100 \mathrm{~s}$. (We denote periods by $U$ for distinction from power $P$.) The wave spectra driving the atmospheric motions were based on the theory of sound generation by turbulent flows (Lighthill 1952; Stein 1967; see also the review by Stein et al. 2004).

Somewhat different simulations were performed by Carlsson \& Stein $(1995,1997)$. They used sequences of measured velocities, not containing short-period waves (for reasons discussed below), to model sub-photospheric velocities at the lower boundaries of the atmosphere. This way, the observed evolution of the $\mathrm{Ca}$ II $\mathrm{H}$ line was successfully reproduced, with brightness fluctuations and asymmetries in and around the line core. The simulated chromospheric dynamics exhibit large temperature excursions but, on time average, no temperature rise outwards. Their conclusion, since then widely embraced, was that, actually, a chromospheric temperature rise does not exist in nonmagnetic areas. However, this would conflict with the observation that UV lines originating in the solar chromosphere are in emission everywhere and all of the time (Carlsson et al. 1997; see also Kalkofen et al. 1999). It is thus important to find out whether short-period waves do play a role in chromospheric heating.

From the observational point of view, it is extremely difficult to detect the short-period waves for two reasons. i) The contribution functions for the velocity/temperature information extend over a height range of at least $200 \mathrm{~km}$, which severely decreases any wave signal with periods shorter than $\sim 60 \mathrm{~s}$. ii) The waves are expected to have small horizontal extension well below 1 arcsec. During the last decade, efforts were undertaken to overcome the observational obstacles.

Fossum \& Carlsson (2005a,b, 2006) used time sequences in the continuum bands at $1600 \AA$ and $1700 \AA$ taken with TRACE (Handy et al. 1999). The spatial resolution of these data is $\sim 1^{\prime \prime}$. Comparison with numerical simulations gave an energy flux in 
the short-period range of at least a factor of 10 too low to account for the chromospheric radiative losses. Straus et al. (2008), among other observations, obtained high-resolution measurements in the Fe I $7090 \AA$ line with the two-dimensional spectrometer IBIS (Reardon \& Cavallini 2008). This line is formed at a height of approximately $250 \mathrm{~km}$. Also Straus et al. did not find enough short-period wave power but sufficient energy flux in gravity waves to balance the chromospheric radiative losses.

A reassessment of the chromospheric heating by shortperiod waves was given by Ulmschneider et al. (2005). They argue that one-dimensional simulations with wave spectra are inadequate since shock merging destroys much of the shortperod waves. They were able to produce a reasonable solar chromospheric model with a monochromatic wave of $20 \mathrm{~s}$ period and a realistic amount of acoustic flux. The analysis of TRACE data by Fossum \& Carlsson (2005a,b, 2006) was criticised by Cuntz et al. (2007) because of the low spatial resolution. Wedemeyer-Böhm et al. (2007) demonstrate with three-dimensional numerical simulations that measurements with too low spatial resolution easily miss a factor of 10 in the short-period energy flux. Thus Carlsson et al. (2007) analysed high-resolution time sequences of $\mathrm{Ca}$ II $\mathrm{H}$ filtergrams from HINODE's SOT (Tsuneta et al. 2008) and concluded that the high-frequency acoustic power in the quiet chromosphere is only $800 \mathrm{~W} \mathrm{~m}^{-2}$, too low to balance the chromospheric radiative losses. Yet the contribution function in their measurements, with a maximum around $200 \mathrm{~km}$ of height, is very extended. Wunnenberg et al. (2002), in a wavelet analysis of data in the Fe I $5434 \AA$ line obtained with a two-dimensional spectrometer, demonstrated the presence of short-period waves and of their strong spatial and temporal intermittency. We further refer to the recent work by Vecchio et al. (2009), and the rich literature cited therein.

The aim of the present contribution is to study waves with observations from ground with high spatial resolution. In view of the diverse observational and simulation results so far, we consider it worth to take such an investigation up again. Here we present new observations obtained with the two-dimensional "Göttingen" spectrometer based on Fabry-Perot interferometers (FPI, Bendlin et al. 1992; Bendlin \& Volkmer 1995; Volkmer et al. 1995), which was substantially improved during the past few years (Puschmann et al. 2006; Bello González \& Kneer 2008). We describe in the following Sect. 2 the observations and the data analysis. We then comment on the properties of the data in terms of response functions and transmission of the solar atmosphere in Sect. 3. The results of Fourier and wavelet analyses will be discussed in Sect. 4. Section 5 concludes the paper.

\section{Observations and data analysis}

\subsection{Observations}

The observations for this study were taken on August 1, 2007, from quiet disc centre of the Sun. We used the Göttingen FPI spectrometer/polarimeter with its upgrades (Puschmann et al. 2006; Bello González \& Kneer 2008) at the VTT/Observatorio del Teide/Tenerife. The observations were supported by the Kiepenheuer Adaptive Optics System (KAOS, von der Lühe et al. 2003). The non-magnetic Fe I $5576 \AA$ line was scanned with the spectrometer, thus the polarimeter of the system was dismounted. The pre-filter for the FPI spectrometer, with central transmission of 0.7 , had a $F W H M$ of $11 \AA$. Time sequences of $\sim 22$ min duration were taken, the Fried parameter varied during the observations between $r_{0}=10 \mathrm{~cm}$ and $r_{0}=20 \mathrm{~cm}$. The reduction of the area to be read out from the CCDs led to a scanning time of $15.8 \mathrm{~s}$. The field of view (FOV) is still appreciable, $\sim 77^{\prime \prime} \times 29^{\prime \prime}$, with a pixel size corresponding to 0.'109. 8 frames were recorded at each of 32 wavelength positions in the line, with wavelength separation of $16.04 \mathrm{~m} \AA$ and exposure times of $15 \mathrm{~ms}$.

From the sampling, the ranges in temporal and spatial frequency result as $v_{\text {Nyquist }}=34 \mathrm{mHz}$ and $k_{\text {Nyquist }}=2 \pi /(2 \Delta x) \approx$ $39.8 \mathrm{Mm}^{-1}$, respectively. The resolutions in frequency and wavenumber are $0.81 \mathrm{mHz}$ and $0.375 \mathrm{Mm}^{-1}$, respectively.

For image reconstruction with speckle methods, broadband frames at $5576 \AA$ (through a filter with $F W H M \sim 50 \AA$ ) were recorded strictly simultaneously with the narrowband spectrometric frames. Furthermore, dark frames and flat field frames with varying pointing of the telescope and with defocussed telescope were taken. Finally, images from a continuum light source (halogen lamp) were obtained which serve to measure the wavelength dependence of the transmission of the spectrometer.

\subsection{Data analysis}

Dark corrections and flat fielding were applied. The broadband frames were reconstructed with the "Göttingen" speckle code (de Boer 1996), which uses the spectral ratio method (von der Lühe 1984) and speckle masking (Weigelt 1977). The speckle reconstruction takes into account that the image correction by KAOS varies with distance from the AO lock point. Thus, the variation of the Fried parameter across the field of view is fitted by a Gaussian centred in the lock point. Such distribution is then used to apply the proper speckle transfer function to each isoplanatic patch (cf. Bello González et al. 2007).

Given the reconstructed broadband image, the shortexposure broadband and simultaneously taken narrowband frames, i.e. the eight frames at each wavelength position, the reconstruction of the narrowband images follows along the lines described repeatedly (see e.g., Keller \& von der Lühe 1992; Krieg et al. 1999; Bello González et al. 2005). After reconstruction, the narrowband images were subjected to a noise (or lowpass) filter with a cutoff corresponding to $0 . ' 3$. The angular resolution in the narrow-band data is estimated from the width of the smallest structures to be in the range of $0 .{ }^{\prime} 4-0.5$. The resolution of the broadband images is approximately 0.25 .

The line profiles at each pixel (and temporal position) were smoothed with a wavelength filter (see Appendix A). Then we determined line minimum intensities $I_{\text {min }}$ and their Doppler shifts from the parabola through the three intensities around the measured line minimum. Wavelength positions of the bisectors at various line depressions were also determined, but are not used here.

The images in the time sequences of the resulting data were shifted to pixel accuracy to maximum correlation of each broadband image to its temporally previous image. The shifts calculated from the broadband images were applied as well to the narrowband data, i.e. velocities and intensities. Then a de-stretching of the broadband time sequence was performed with the code of Yi \& Molowny Horas (1992). For the de-streching we require best agreement of the instantaneous broadband image to the average of the 11 temporally closest images. And again, the destreching parameters for the broadband sequence were applied to the other time series.

We restrict the further analysis and discussion to the central subfield of the full FOV of $23^{\prime \prime} \times 23^{\prime \prime}$ around the lock point of the AO. Figure 1 shows a snapshot from the broadband images, 

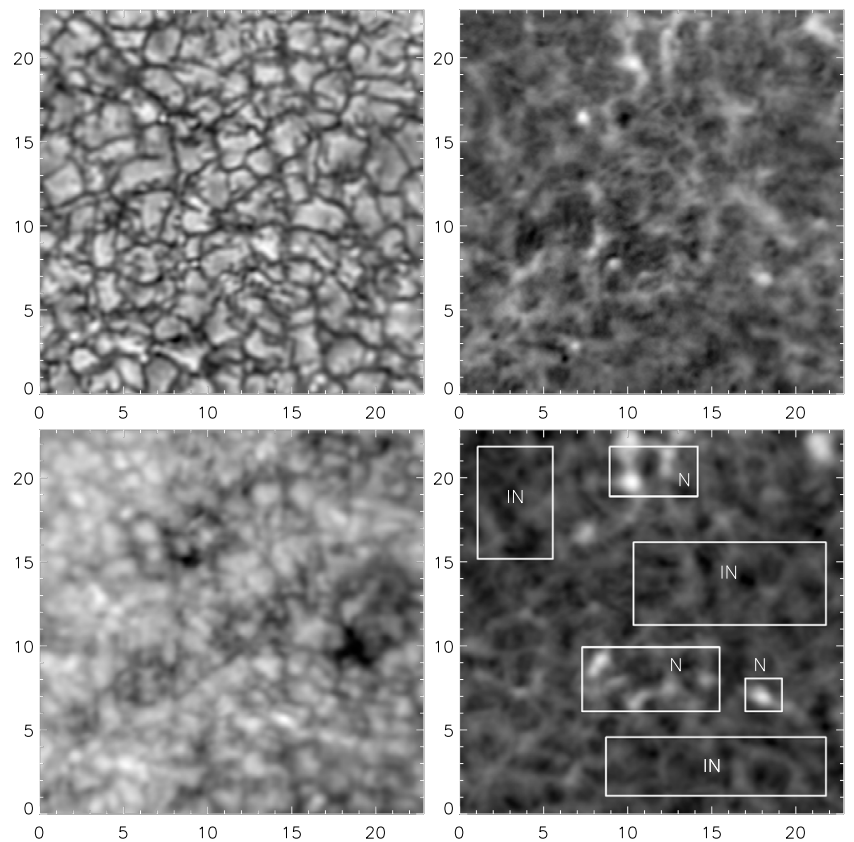

Fig. 1. From time series in Fe I $5576 \AA$ line, clockwise starting at $u p$ per left: broadband image, minimum intensity, minimum intensity averaged over time sequence, and velocity from minimum position of line with bright indicating upward flow. The boxes with " $\mathrm{N}$ " and "IN" indicate network and inter-network areas, respectively. Axes are in arcsec. The dark patches in the velocity map (lower left) at positions $(20,10)$ and $(7,15)$, result from the coincidence of intergranular down-flow and the downwards motions from the 5-min osciallations.

from the line minimum images $I_{\min }$, from the line minimum velocities $v_{\min }$, and the line minimum intensity averaged over the time sequence. The broadband image has a spatial resolution of $\sim 0$ '.25, the line minimum and velocity snapshots have $0 .{ }^{\prime} 4-0 . ' 5$ resolution. The 5-min oscillations were not removed in the data for Fig. 1, e.g. by high-pass filtering (but see Sect. 4.1). So these and the granular overshoot dominate the appearance of the velocity images. The rms fluctuations are $I_{\mathrm{bb}, \mathrm{rms}} /\left\langle I_{\mathrm{bb}}\right\rangle=0.097$ for the broadband sequence, $I_{\min , \mathrm{rms}} /\left\langle I_{\min }\right\rangle=0.14$ for line minimum, and $v_{\text {min,rms }}=0.41 \mathrm{~km} \mathrm{~s}^{-1}$ for the velocity at line minimum.

In the average line minimum image, we marked areas of network and pure inter-network by "N" and "IN", respectively. We excluded areas close to the borders, since the Fourier analysis and high-pass filtering will need apodisation. The network is recognisable by enhanced brightness in line minimum, caused by the reduced opacities in small-scale magnetic flux tubes (e.g., Bello González et al. 2007). The study of wave behaviour in the network, in comparison with inter-network, is of interest to distinguish between possible heating mechanisms in areas with strong and weak magnetic flux. Atmospheric oscillations along solar magnetic flux tubes were treated analytically and numerically by Musielak \& Ulmschneider (e.g., 2003a,b). Twodimensional numerical simulations in small-scale flux tubes with strong fields were carried out by Hasan et al. (2005) and Hasan \& van Ballegooijen (2008).

\section{Response functions and atmospheric transmission}

\subsection{Response functions}

According to Shchukina \& Trujillo Bueno (2001), the Fe I 5576 line has line centre optical depth $\tau_{\text {lc }}=1$ at a height

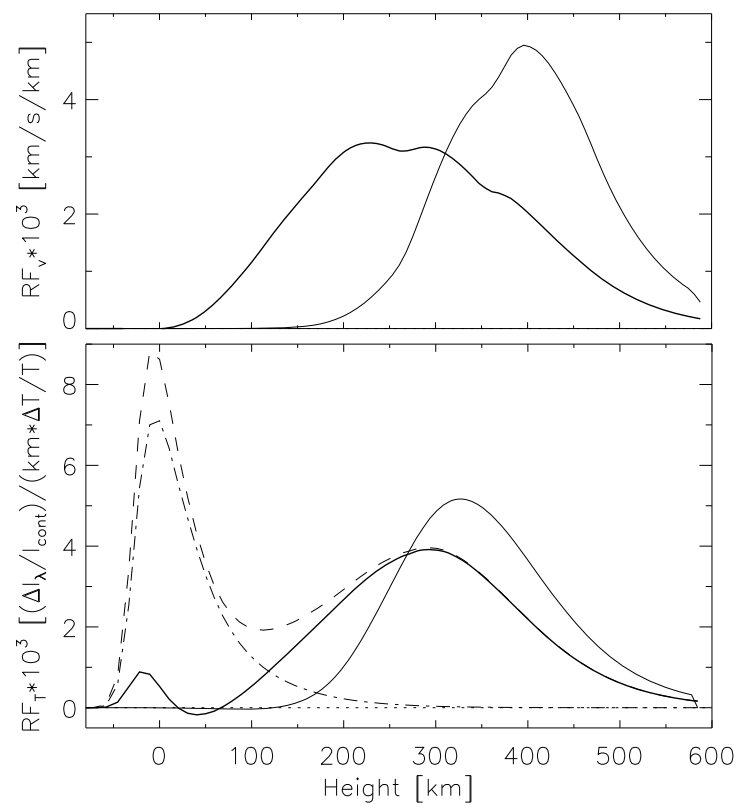

Fig. 2. Upper panel: response functions for velocity $R F_{v}(z)$ from line minimum with infinite spectral resolution (solid) and after convolution with spectrometer function and filtering (thick solid); lower panel: temperature response functions $R F_{T}(z)$ for line minimum intensity with infinite spectral resolution (thin solid), same after convolution and filtering in wavelength (dashed), of continuum intensity scaled down by a factor of 6 (dash-dotted), and linear combination following Eq. (1) (thick solid).

of $400 \mathrm{~km}$ in granules and at $420 \mathrm{~km}$ in inter-granules. We calculated response functions of intensity fluctuations from fluctuations of atmospheric parameters (see Mein 1971). We present response functions for the velocity causing Doppler shifts of the line minima, $R F_{v}(z)$, and for the temperature fluctuations causing the intensity fluctuations at the line minima, $R F_{T}(z)$. The calculations were done for this Fe I 5576 line in the VALC model (Vernazza et al. 1981), assuming LTE. The method described by Eibe et al. (2001) was applied. For the present purposes, only the effect of temperature fluctuations on the source function, or the Planck function in LTE, was treated, not the effect on the opacity.

Figure 2 depicts the response functions for velocity (upper panel) and for temperature (lower panel). If we assume a perfect spectrometer, i.e. infinite spectral resolution, $R F_{v}(z)$ peaks at a height of about $400 \mathrm{~km}$ (thin solid curve in upper panel of Fig. 2), at the same height as from the multidimensional nonLTE calculations by Shchukina \& Trujillo Bueno (2001). Yet applying to the line intensities which emerge from the disturbed VALC model atmosphere the same spectrometric broadening and filtering in wavelength as to the observed data, we obtain the thick solid line in Fig. 2, upper panel. Due to mixing of intensities around the line minimum, the response function has become broader and has moved down by approximately $150 \mathrm{~km}$ compared to the original one.

In our measurements, the average profile of the Fe I $5576 \AA$ line had a minimum intensity approximately a factor of two higher than the one from the Fourier Transform Spectrometer Atlas (FTS, Brault \& Neckel, quoted by Neckel 1999). This is due partly to the method of data acquisition and analysis, i.e. finite spectral resolution and wavelength filtering, but mostly to the use of a broad pre-filter which admits much intensities 
from neighbouring wavelengths. The details are discussed in Appendix A.

To obtain information on the formation height of the intensity fluctuations at line minimum, we calculated also the temperature response functions depicted in the lower panel of Fig. 2. For infinite spectral resolution we get the thin solid curve, which peaks at approximately $330 \mathrm{~km}$. Applying the convolution with the spectrometric profile and wavelength filtering we see again a broadening and shift to lower heights (dashed curve). Yet in addition, a large contribution from low heights is added. This results from the intensities in a broad, not fully suppressed, essentially continuum wavelength band through the pre-filter.

For a "cleaner" response function we can partly eliminate the contribution from deep layers using a linear combination of response functions as

$R F_{T, \min }(z)=R F_{T, \min , \mathrm{obs}}(z)-0.19 * R F_{T, \text { cont }}(z)$,

where $R F_{T \text {,min,obs }}(z)$ denotes the temperature response function simulating the observations and $R F_{T \text {,cont }}(z)$ is the response function for the continuum, which is shown by the dash-dotted curve in Fig. 2 after down-scaling by a factor of 6 . The resulting response function $R F_{T \text {,min }}(z)$ is given by the thick solid curve in the lower panel of Fig. 2.

The line minimum intensities in Fig. 1 were treated according to Eq. (1). We note that this process does not affect the results of the wavelet analysis below (Sect. 4.2). Also, the mixing of mainly continuum intensities into the line intensities has no influence on the Doppler shifts of the spectral line.

\subsection{Atmospheric transmission}

It had been pointed out repeatedly that the extent in atmospheric height of the contribution (or response) functions decreases the observable signal of small-scale fluctuations along the line of sight, also of short-period waves (e.g., Durrant 1979; Cram et al. 1979; Mein \& Mein 1980).

We calculated transmissions of velocity amplitudes in the VAL C model atmosphere (Vernazza et al. 1981) in the following way. We assumed velocities of vertically propagating waves with low amplitudes $v_{0}(z)$ with periods from $30 \mathrm{~s}$ to $190 \mathrm{~s}$, at 25 phases through a full period for each wave. To mimic the atmospheric stratification and have the acoustic energy flux approximately constant through the atmosphere we require

$\rho(z) \cdot v_{0}^{2}(z) \approx$ const.

or

$v_{0}(z)=v_{0}(z=0) \cdot \mathrm{e}^{z /(2 H)}$,

with mass density $\rho(z)$ and density scale height $H$ for which we take $110 \mathrm{~km}$. For the propagation speed of the waves we use the phase velocity from the dispersion relation

$k_{z}^{2}=\left(\omega^{2}-\omega_{\mathrm{ac}}^{2}\right) \frac{1}{c_{\mathrm{s}}^{2}}-\left(\omega^{2}-\omega_{\mathrm{BV}}^{2}\right) \frac{k_{h}^{2}}{\omega^{2}}$.

Here, $\omega=2 \pi v$ is the angular frequency, $k_{z}$ and $k_{h}$ are the vertical and horizontal wave numbers, respectively, $\omega_{\mathrm{ac}}$ and $\omega_{\mathrm{BV}}$ the acoustic cutoff and Brunt-Väisälä frequency, respectively, with

$\omega_{\mathrm{ac}}=\frac{\gamma g}{2 c_{\mathrm{s}}}, \quad \omega_{\mathrm{BV}}=\frac{\sqrt{\gamma-1} g}{c_{\mathrm{s}}}$,

where $\gamma=5 / 3$ is the ratio of specific heats, $g$ the surface gravity acceleration, and $c_{\mathrm{s}}$ the sound velocity for which we take

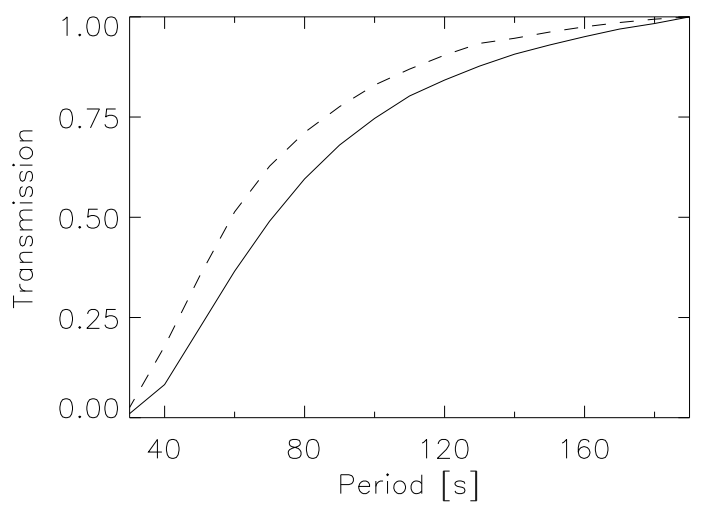

Fig. 3. Transmission of solar atmosphere for acoustic waves as function of wave period with infinite spectral resolution (dashed) and after applying the convolution with spectrometer function and filtering.

$7 \mathrm{~km} \mathrm{~s}^{-1}$. With this the acoustic cutoff frequency is $\omega_{\mathrm{ac}}=3.26 \times$ $10^{-2} \mathrm{~s}^{-1}$ and the cutoff period is $U_{\mathrm{ac}}=193 \mathrm{~s}$.

For vertically propagating waves $\left(k_{h}=0\right)$ the phase velocity is from Eq. (4)

$v_{\mathrm{ph}}=\frac{\omega}{k_{z}}=\frac{c_{\mathrm{s}}}{\sqrt{1-\left(\omega_{\mathrm{ac}} / \omega\right)^{2}}}$

while the group velocity, with which the energy is transported, is

$v_{\mathrm{gr}}=\frac{\mathrm{d} \omega}{\mathrm{d} k_{z}}=c_{\mathrm{s}} \cdot \sqrt{1-\left(\omega_{\mathrm{ac}} / \omega\right)^{2}}$.

Figure 3 gives the transmission of the wave amplitudes by the model atmosphere, i.e. the ratio of velocity amplitude, observable as Doppler shift of the line minimum to true amplitude, as function of period. We assume that for $U=190 \mathrm{~s}$, which is close to the cutoff period, the transmission is 1 . The dashed curve in Fig. 3 results for a spectrometer with infinite resolution, the solid curve is obtained when convolving the emergent line profiles with the spectrometer's profile and filtering in wavelength. The square of the transmission curves as function of period (or frequency) will be used below for correction of the velocity power. It is commonly called the transfer function, $T F(v)$. The observed rms velocity at periods $U$ shorter than the acoustic cutoff period is approximately $0.1 \mathrm{~km} \mathrm{~s}^{-1}$, thus much shorter than the thermal velocity of iron of $\sim 1.2 \mathrm{~km} \mathrm{~s}^{-1}$, which assures the viability of the method of calculating the transmission. We note that for waves with periods of $50 \mathrm{~s}$ the wave power in the measurement is decreased by a factor of 20 , while for a perfect spectrometer the power is decreased by a factor of 8 . On the basis of numerical simulations, Stein et al. (2004, their Fig. 9) point out that, in addition to the signal suppression at short periods, there is increasing radiative damping with decreasing period.

\section{Results and discussion}

We now present results first from the Fourier analysis and subsequently from the wavelet analysis. The implications of the results will then be discussed in Sect. 4.3.

\subsection{Fourier analysis}

The temporal power spectrum from the velocity fluctuations, averaged over the pixels in the FOV, is depicted as the dashed curve in Fig. 4. It shows the signatures of granular motions at around $0.5-2 \mathrm{mHz}$ and of the 5 -min oscillations from $2 \mathrm{mHz}$ 

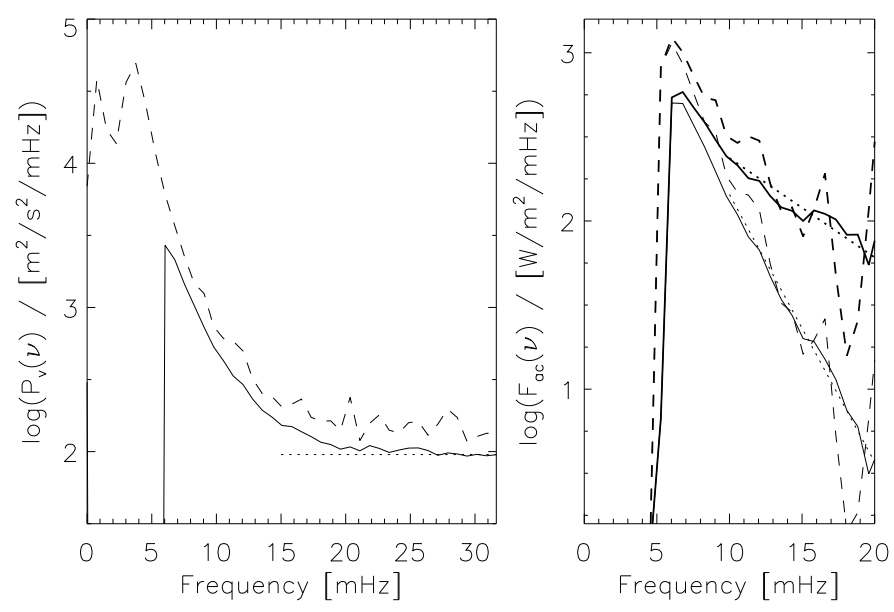

Fig. 4. Left panel: averaged temporal power spectra $P_{v}(v)$ from line minimum velocities; dashed: original data, solid: after high-pass filtering in $k_{h}$ and $v$ (see text); horizontal dotted line: noise level. Right panel: acoustic energy flux spectra $F_{\text {ac }}(v)$ from the velocity power spectra; dashed thin: from original data, dashed thick: from original data after correction for atmospheric transmission (cf. Fig. 3 and Eq. (8)); solid thin: from high-pass filtered data without correction, solid thick: same after correction; dotted lines: slopes for extrapolation.

to $5 \mathrm{mHz}$ and beyond. The (average) power spectra reaches the noise level at approximately $25 \mathrm{mHz}$. The two-dimensional power spectrum $P_{v}\left(k_{h}, v\right)$ (not presented), with horizontal wave number $k_{h}=\left(k_{x}^{2}+k_{y}^{2}\right)^{1 / 2}$, shows that the noise resides mainly close to $k_{h}=0$ along the frequency axis. It results from small residual jitter in the data.

We have reduced this part of the noise by applying a highpass filter to the three-dimensional Fourier transforms of the data. The amplitudes were set to zero at wavelengths larger than $10^{\prime \prime}$. At the same time we set also to zero the amplitudes outside the domain of propagation of acoustic waves in the $k_{h}-v$ diagram, i.e. where $k_{z}^{2}<0$ and $\omega<\omega_{\mathrm{ac}}$ in Eq. (4) above. The resulting temporal power spectrum is given as the solid curve in Fig. 4. The horizontal dotted line in this figure gives our estimate of the noise level in the high-pass filtered power spectrum. We shall restrict the further analyses and discussion mainly to frequencies $\leq 20 \mathrm{mHz}$.

After subtraction of the noise level from the power spectra we may now estimate the acoustic flux spectra and the total flux at the layer where the velocity signal is formed. We use the formulae

$F_{\mathrm{ac}}\left(v_{i}\right)=\rho \cdot P_{v}\left(v_{i}\right) \cdot v_{\mathrm{gr}}\left(v_{i}\right) / T F\left(v_{i}\right)$

for the flux spectrum, with mass density $\rho$ at the level of formation, and

$F_{\text {ac,tot }}=\sum_{i} F_{\text {ac }}\left(v_{i}\right) \cdot \Delta v_{i}$

for the total acoustic flux with summation over frequency intervals $\Delta v_{i}$. Dividing in Eq. (8) by the transfer function $T F(v)$ of the atmosphere includes the correction of the flux spectra (cf. Sect. 3.2). For the density we adopt the value $\rho=5.4 \times$ $10^{-5} \mathrm{~kg} \mathrm{~m}^{-3}$ at a height of $250 \mathrm{~km}$ in the VAL C model (Vernazza et al. 1981).

The flux spectra are shown in the right panel of Fig. 4. The thin and thick dashed curves give the spectra before and after correction for the atmospheric transmission, respectively. The thin and thick solid curves are those for the high-pass filtered data.
The dotted straight lines in the right panel of Fig. 4 indicate the frequency dependences of the acoustic flux spectra. With these we may extrapolate to higher frequencies and estimate the energy flux carried by acoustic waves with periods $U<50 \mathrm{~s}$ (cf. Sect. 4.3).

The study of pure inter-network and network areas, as indicated in Fig. 1, gave no large difference in velocity power. We shall discuss in detail the amounts of flux below in Sect. 4.3 together with those found from the wavelet analysis. But we note here that we obtain from the high-pass filtered data flux values of $1840 \mathrm{~W} \mathrm{~m}^{-2}$ and $3190 \mathrm{~W} \mathrm{~m}^{-2}$ before and after correction, respectively.

\subsection{Wavelet analysis}

The temporal power spectra averaged over the FOV (cf. Fig. 4, left panel) do not show structure, apart from the signature of the 5-min oscillations. We therefore perform a wavelet analysis as in Wunnenberg et al. (2002). The code by Torrence \& Compo (1988) was used with Morlet wavelets. This allows us to localise at each pixel the occurrence of velocity and intensity fluctuations along the time sequences and to determine their amplitude. We calculated the wave power in the period bands $50 \mathrm{~s}<U<70 \mathrm{~s}$, $70 \mathrm{~s}<U<90 \mathrm{~s}, \ldots$, up to $170 \mathrm{~s}<U<190 \mathrm{~s}$. A statistical significance level of $95 \%$ was adopted.

Figures 5 and 6 give examples of results from the wavelet analysis of velocity and intensity at line minimum. The brightness levels indicate the square of the fluctuation. They are inverted for better visualisation, i.e. the darker the grey scale the more power was found. The high-pass filtered data were used for these figures. Since filtering requires apodisation in spatial (and temporal) coordinates, the FOV is reduced to $20.7 \times 20 ! 7$.

Figure 5 shows the position of power in the period range $U=(140 \pm 10) \mathrm{s}$. One sees from left to right: velocity power at a specific time $t \approx 300 \mathrm{~s}$ after the start of observation, intensity power at the same time, velocity power averaged over time, and intensity power averaged over time. We note from inspection of this figure and of the according full time sequence:

1. The power is produced in patches of $\sim 1^{\prime \prime}$, rarely larger than $2^{\prime \prime}$ size. Acoustic flux appears and disappears simultaneously in the power maps of both velocity and intensity.

2. The power patches last for $(5 \pm 2)$ min and often move horizontally with velocities of the order of $15 \mathrm{~km} \mathrm{~s}^{-1}$.

3 . Velocity and intensity power maps possess similar morphology, i.e. appear to be coherent, but do not agree in detail such as in the temporal development of amplitude.

4. The power in the periods of $U=(140 \pm 10) \mathrm{s}$ is intermittent. This agrees with the finding of Vecchio et al. (2009) from the chromospheric Ca II $8542 \AA$ line that shocks in this period range are abundant but occur in rare bursts. Even after temporally averaging over the $19.2 \mathrm{~min}$ of the sequence (two right panels of Fig. 5) one still has variations by a factor of 10 between minimum and maximum power in the FOV.

In this period range, the signal comes from propagating acoustic waves which show, in the $k_{h}-v$ power spectra, the ridges of the pseudo-p-modes (cf. Krijger et al. 2001, their Fig. 23, and references therein). The horizontal phase velocity $v_{\mathrm{ph}, h}=\omega / k_{h}$ of such waves can be large.

Figure 6 exhibits power maps in the period bin $U=(80 \pm$ 10) $\mathrm{s}$, with velocity power in the upper row and intensity power in the lower row. We present from left to right: power maps at $t \approx 300 \mathrm{~s}$ after the start of the sequence, at $t \approx 727 \mathrm{~s}$, and the 

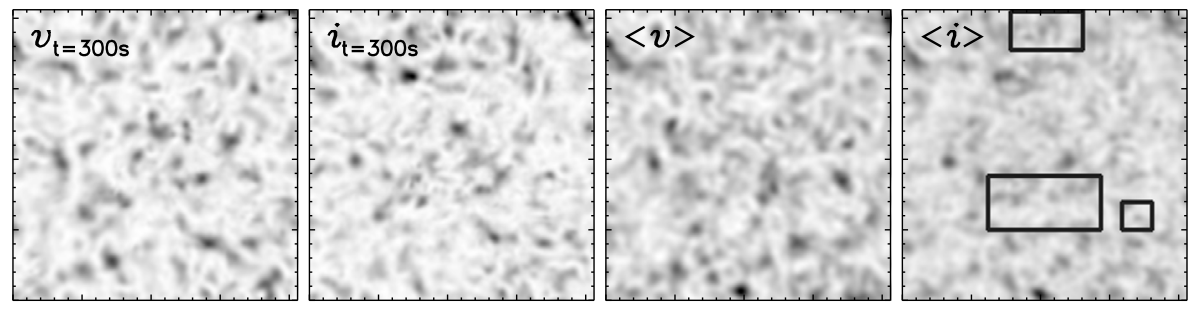

Fig. 5. Spatial position of power of acoustic waves in the period range 130-150 s. From left to right: velocity power at $t \approx 300 \mathrm{~s}$ after the start of observation, intensity power at the same time, velocity power averaged over time, and intensity power averaged over time. The grey scales of the power levels are inverted, i.e. high power is represented dark; tick marks at $1^{\prime \prime}$ distance. The rectangles in the rightmost power map denote the network areas from Fig. 1.

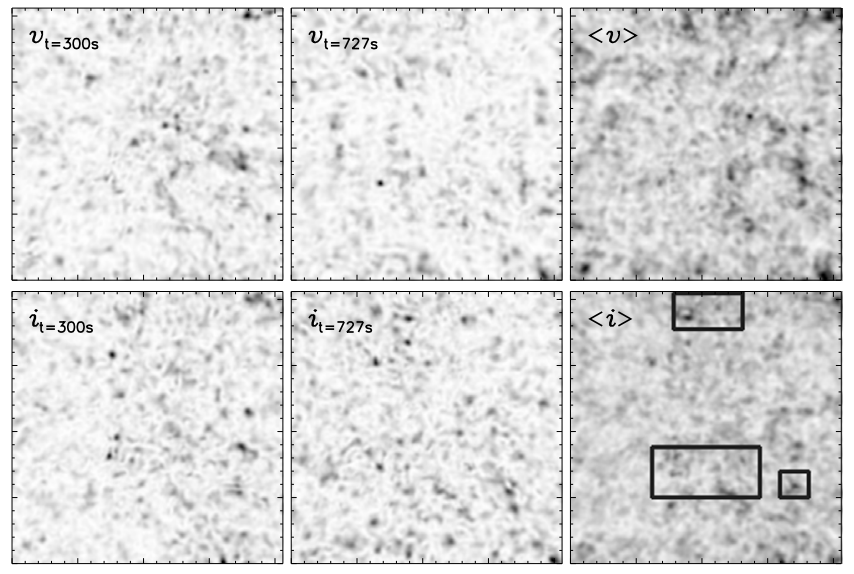

Fig. 6. Spatial position of power in the period bin $U=(80 \pm 10) \mathrm{s}$ from velocities (upper row) and intensities (lower row). From left to right: at $t \approx 300 \mathrm{~s}$, at $t \approx 727 \mathrm{~s}$, and the temporal averages. Grey scales inverted, tick marks at $1^{\prime \prime}$ distance. The rectangles in the lower right power map denote the network areas from Fig. 1.

temporal averages. We note from this figure and from inspection of the movies along the time sequences:

1. The signals are very intermittent in space and time, the waves are of small scale, mostly well below 1 arcsec.

2. The power lasts often for only $2.5-4 \mathrm{~min}$, i.e. not more than 2 to 3 periods.

3. We see extended patches where waves at this period bin are preferentially produced. There the velocity power reappears with large amplitude after some 5-10 $\mathrm{min}$ at the same or at a nearby location. The patches of the velocity wavelets are not co-spatial with network areas, cf. Fig. 1. But the wavelets from the line minimum intensity fluctuations show similarity with network structure in some cases.

4. In many cases, strong signals lasting for approximately $150 \mathrm{~s}$ in the velocity measurements are followed, in the same area, by similar signals in line minimum intensity fluctuation, shifted by $15-30 \mathrm{~s}$ and with slightly different spatiotemporal morphology.

5. The agreement between the velocity and line minimum intensity power is not as good as for the $(140 \pm 10) \mathrm{s}$ period bin. But the areas of power production agree coarsely.

6. In ample areas no power appears during the $19.2 \mathrm{~min}$ of this time sequence.

The dependence of short-period energy in the line minimum velocities on the granular convection is depicted in Fig. 7. There the counts of wavelet power for the periods $110 \mathrm{~s}<U<190 \mathrm{~s}$ (solid) and $50 \mathrm{~s}<U<110 \mathrm{~s}$ (dashed) vs. the normalised broadband intensity $I_{\mathrm{bb}}(t-31.6 \mathrm{~s}) /\left\langle I_{\mathrm{bb}}\right\rangle$ at each position in the FOV are presented. The broadband images were taken two time steps $(=31.6 \mathrm{~s})$ earlier than the wavelet powers under analysis. This time lap corresponds approximately to the travel time from the

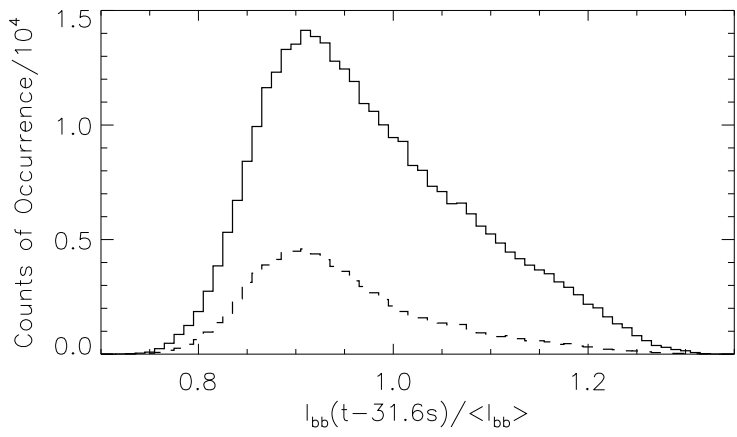

Fig. 7. Occurrence of wavelet power of velocities vs. normalised broadband intensity $I_{\mathrm{bb}}(t-31.6 \mathrm{~s}) /\left\langle I_{\mathrm{bb}}\right\rangle$ at two time steps earlier than the measurement of the velocities. Solid: in period range 110-190 s, dashed: in period range $50-110 \mathrm{~s}$.

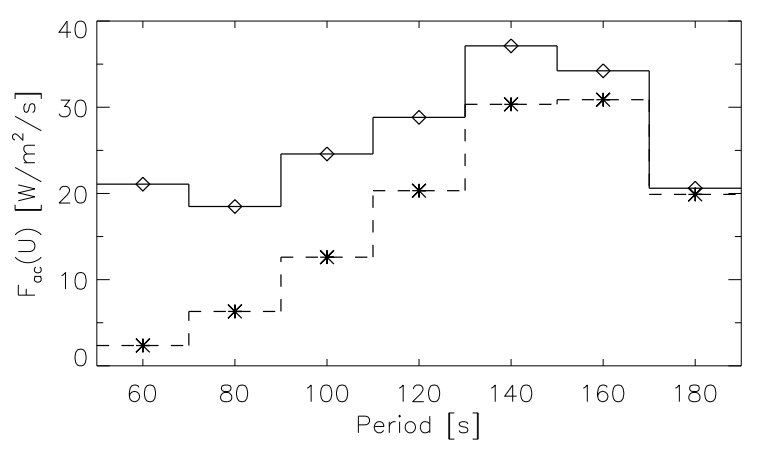

Fig. 8. Energy flux in period bins 50-70 s, ..., 170-190 s from wavelet analysis. Dashed and asterisks: uncorrected for atmospheric transmission; solid and rhombs: corrected.

bottom of the photosphere to $250 \mathrm{~km}$ height $\left(\sim 250 \mathrm{~km} / v_{\mathrm{ph}}\right)$. For the longer periods, we collected the positions in time and space where the power is larger than a factor of 0.3 of the maxima in each period band of width $\Delta U=20 \mathrm{~s}$. For the shorter periods a threshold of 0.2 of the corresponding maxima was chosen.

Figure 7 shows clearly that the wave energy is produced predominantly in dark intergranular areas, as already found by Wunnenberg et al. (2002, their Figs. 3 and 4). We note without showing that this behaviour is present in all period bands of $20 \mathrm{~s}$ width, from $50 \mathrm{~s}$ to $190 \mathrm{~s}$. Movies of granular evolution overlaid with power in various period bins confirm this finding. In addition, one also sees wavelet power occurring above small rapidly evolving granules. Wavelet power may also occur above granules before they split and above granular borders.

The results on the acoustic flux distributions averaged over time and over the total FOV, are given in Fig. 8. There we show the dependence on period $U$ for both the uncorrected and corrected values, i.e. without division by $T F_{i}$ and with division. 
The total acoustic flux is obtained, similarly as from Eqs. (8) and (9) above, by

$\left.F_{\text {ac,tot }}=\Delta U \cdot \rho \cdot \sum_{i}<v_{i}^{2}\right\rangle \cdot v_{g r, i} / T F_{i}$,

with summation over the period intervals, and again mass density $\rho=5.4 \times 10^{-5} \mathrm{~kg} \mathrm{~m}^{-3}$ at $z=250 \mathrm{~km}$ in the VALC model. As in the Fourier analysis, we also distinguished between pure inter-network regions and network as indicated in the temporal average image of $I_{\min }$ in Fig. 1. The values for the total acoustic energy fluxes also are given in Table 1. We note here that they are similar to the results from the Fourier analysis, and the total flux averaged over time and the full FOV is $3650 \mathrm{~W} \mathrm{~m}^{-2}$.

Towards short periods, below $80 \mathrm{~s}$, the corrected flux appears to increase, at least to level off at approximately $20 \mathrm{~W} \mathrm{~m}^{-2} \mathrm{~s}^{-1}$ (cf. Fig. 8). Taken such a constant flux level at face value and extrapolating to periods of $10 \mathrm{~s}$ would imply an additional acoustic flux of the order of $800 \mathrm{~W} \mathrm{~m}^{-2}$. We note that extrapolation of the flux spectra from the Fourier analysis to $100 \mathrm{mHz}$ gave an additional flux of up to $400 \mathrm{~W} \mathrm{~m}^{-2}$. Thus, such an amount of flux from extrapolation is not totally improbable. Alternatively, the correction is too large. We shall expand on this point below.

\subsection{Discussion}

We collect the results on acoustic flux from our Fourier and wavelet analyses in Table 1 together with values given by other authors. In this table, "IN" and "N" denote inter-network and network results, respectively and "corr." indicates correction of the flux values for the atmospheric transmission, i.e. division by $T F(v)$ (cf. Sect. 3.2). The lower limit values in italic are from application of smaller correction factors.

The table contains in the rightmost column the total fluxes, i.e. the sums over the frequency range $5.2-20 \mathrm{mHz}$. The correction with the atmospheric transfer function $T F(v)$ is uncertain. $T F(v)$ is based on simulations of observable velocity amplitudes from waves propagating vertically through a quiet Sun model atmosphere. We are confident that the correction factors are reliable at frequencies below $10 \mathrm{mHz}$. But they become increasingly uncertain with increasing frequency.

We estimated the lower limits in Table 1 of the acoustic fluxes in the various frequency bands by applying the transmission of a perfect spectrometer, i.e. the dashed transmission function in Fig. 3. The height of signal formation, $h=250 \mathrm{~km}$, was not changed for the estimate. This yields, compared with the "fully" corrected values, a reduction by $10 \%$ in the $5.2-10 \mathrm{mHz}$ band and by $40 \%$ for $10-20 \mathrm{mHz}$, and the flux from extrapolation beyond $20 \mathrm{mHz}$ has become $110 \mathrm{~W} \mathrm{~m}^{-2}$.

In the wavelet analysis, we assigned half of the flux in the period bin of $90-110 \mathrm{~s}$ to the $5.2-10 \mathrm{mHz}$ frequency band and the other half to the $10-20 \mathrm{mHz}$ band. This analysis gave similar acoustic fluxes as the Fourier analysis. The values in the $5.2-10 \mathrm{mHz}$ band are higher than those from the Fourier analysis by approximately $30 \%$, while the values at $10-20 \mathrm{mHz}$ are lower by $10-20 \%$. Also here, no significant differences between inter-network and network are seen.

We discuss shortly extrapolations of the fluxes towards frequencies higher than $20 \mathrm{mHz}$. We emphasise that such extrapolated values are speculative. However, we realise that our observations both with Fourier and wavelet analysis indicate additional acoustic flux at higher frequencies. Extrapolating the slopes of the flux spectra in Fig. 4, right panel we may integrate and obtain from the Fourier analysis additional acoustic flux in
Table 1. Energy fluxes in acoustic waves $F_{\text {ac }}$ from quiet Sun disc centre. See text for meaning of "IN", "N", "corr.", and lower limit.

\begin{tabular}{|c|c|c|c|}
\hline \multirow[t]{2}{*}{ comment } & \multicolumn{3}{|c|}{$F_{\mathrm{ac}}\left[\mathrm{W} \mathrm{m}^{-2}\right]$} \\
\hline & $5.2-10 \mathrm{mHz}$ & $10-20 \mathrm{mHz}$ & total \\
\hline \multicolumn{4}{|l|}{ Fourier analysis } \\
\hline full FOV & 1500 & 340 & 1840 \\
\hline full FOV, corr. & 1910 & 1280 & 3190 \\
\hline lower limits & 1720 & 780 & 2500 \\
\hline IN, corr. & 2030 & 1370 & 3400 \\
\hline $\mathrm{N}$, corr. & 1860 & 1270 & 3130 \\
\hline \multicolumn{4}{|l|}{ wavelet analysis } \\
\hline full FOV & 2150 & 310 & 2460 \\
\hline full FOV, corr. & 2630 & 1020 & 3650 \\
\hline lower limits & 2410 & 630 & 3040 \\
\hline IN, corr. & 2710 & 990 & 3700 \\
\hline $\mathrm{N}$, corr. & 2710 & 1150 & 3860 \\
\hline \multicolumn{3}{|l|}{ other work } & total \\
\hline \multicolumn{3}{|c|}{$\begin{array}{l}\text { Wunnenberg et al. (2002) } \\
\text { acoustic waves, } 7.7-20 \mathrm{mHz} \text { at } h=600 \mathrm{~km}\end{array}$} & 900 \\
\hline \multicolumn{4}{|c|}{ Straus et al. (2008) } \\
\hline \multicolumn{3}{|c|}{ acoustic waves at $h=250 \mathrm{~km}$} & 1400 \\
\hline \multicolumn{3}{|c|}{ acoustic waves at $h=500 \mathrm{~km}$} & 1000 \\
\hline \multicolumn{3}{|c|}{ gravity waves at $h=250 \mathrm{~km}$} & 20800 \\
\hline \multicolumn{3}{|c|}{ gravity waves at $h=500 \mathrm{~km}$} & 5000 \\
\hline \multicolumn{3}{|c|}{ Fossum \& Carlsson (2006), acoustic waves } & 510 \\
\hline \multicolumn{3}{|c|}{ Carlsson et al. (2007), acoustic waves } & 800 \\
\hline
\end{tabular}

the range $20-100 \mathrm{mHz}$ of $10 \mathrm{~W} \mathrm{~m}^{-2}$ for the case of uncorrected fluxes. The extrapolation of the flux distribution corrected for atmospheric transmission gives additional power of $450 \mathrm{~W} \mathrm{~m}^{-2}$ and $110 \mathrm{~W} \mathrm{~m}^{-2}$ for the extrapolation in the lower limit case. For the wavelet analysis, due to lack of better observational knowledge, we extrapolate the period dependence down to $10 \mathrm{~s}$ with constant flux distribution using the values at the $60 \mathrm{~s}$ period. This gives for the uncorrected flux in the $20-100 \mathrm{mHz}$ range an additional flux of $110 \mathrm{~W} \mathrm{~m}^{-2}$ and for the flux corrected for atmospheric transmission $840 \mathrm{~W} \mathrm{~m}^{-2}$. This appears to be large, but we consider it not improbable. In the lower limit case we obtain additional $380 \mathrm{~W} \mathrm{~m}^{-2}$.

Jefferies et al. (2006) have pointed out that strong magnetic fields at the supergranular boundaries open a "portal" for propagation of magnetoacoustic waves with periods longer than the acoustic cutoff period ( $190 \mathrm{~s}$ ). This has not been accounted for in our analyses. Obviously, the magnetic chromosphere needs more energy supply for the balance of the radiative losses. In the present study we search for the basal energy flux into those parts of the solar chromosphere which show very low magnetic activity (Schrijver 1987).

The total acoustic flux found here from velocity fluctuations amounts to $\sim 3000 \mathrm{~W} \mathrm{~m}^{-2}$ at a height of $h=250 \mathrm{~km}$ above $\tau_{5}=1$ in the VALC atmospheric model (Vernazza et al. 1981). We also find that $65-75 \%$ of the flux is carried by waves with frequencies of $5.2-10 \mathrm{mHz}$ and $25-30 \%$ by waves with higher frequencies. Wunnenberg et al. (2002) measured, also in a wavelet analysis, yet without correction for atmospheric transmission, an acoustic flux of $900 \mathrm{~W} \mathrm{~m}^{-2}$ in the period range $7.7-20 \mathrm{mHz}$ at $h \approx 600 \mathrm{~km}$. The total flux from the present study is higher by a factor of approximately 2 than the value of $1400 \mathrm{~W} \mathrm{~m}^{-2}$ given by Straus et al. (2008) at the same atmospheric height. This latter value is not corrected for atmospheric transmission. It is somewhat lower than the 1850 and $2500 \mathrm{~W} \mathrm{~m}^{-2}$ for our uncorrected measurements from Fourier and wavelet analysis, respectively. We speculate that the spatial resolution in the present work is 
somewhat higher than in the data used by Straus et al. According to the numerical simulations by Straus et al. (2008), the flux would decrease by $\sim 30 \%$ before reaching the base of the average chromosphere at $h \approx 500 \mathrm{~km}$ due to radiative damping. Yet the actual reduction is again uncertain (cf. Fig. 3 in Straus et al. 2008). The high frequency waves are the most prone to damping (Stein et al. 2004). Also, it is important to note that the flux measurements by Straus et al. (2008) are in excellent agreement with the results of their numerical simulations. This underlines the needs for further work in this field, combining high-resolution observations with sophisticated numerical simulations.

An interesting new finding on energy flux carried by atmospheric gravity waves was reported by Straus et al. (2008). They give a flux of $20800 \mathrm{~W} \mathrm{~m}^{-2}$ at $h=250 \mathrm{~km}$, which is reduced to $\sim 5000 \mathrm{~W} \mathrm{~m}^{-2}$ at $h=500 \mathrm{~km}$, an amount of energy supply to account for the heating of atmospheric layers above that height to a "warm" chromosphere from which UV emission lines would be observed. Vernazza et al. (1981) give radiative losses of $4600 \mathrm{~W} \mathrm{~m}^{-2}$ for their model C, while Anderson \& Athay (1989) quote $14000 \mathrm{~W} \mathrm{~m}^{-2}$ when including radiative losses from Fe II lines.

The energy flux in acoustic waves found by Fossum \& Carlsson (2006) from intensity fluctuations in TRACE filtergram time sequences is much below the flux found here, by a factor of 6 , at least by a factor of 4 when accounting for possible wave damping for our result at $h=250 \mathrm{~km}$. Likewise, the measurement of intensity fluctuations with high-spatial resolution from HINODE filtergram time series in Ca II H by Carlsson et al. (2007) gave acoustic fluxes lower than a factor of 3-4 than our result. We suggest that the TRACE data have not sufficient spatial resolution to detect more short-period waves, while the HINODE filtergrams in Ca II $\mathrm{H}$ measure over a too extended height range.

\section{Conclusions}

We have analysed a time series of two-dimensional (2D) narrowband spectrograms in the non-magnetic Fe I $5576 \AA$ line. The sequence had a duration of $\sim 22 \mathrm{~min}$ and a cadence of $15.8 \mathrm{~s}$. The data were reconstructed with speckle methods. Intensity and velocity fluctuations at line minimum have been determined, and the velocities were corrected for the transmission of the solar atmosphere. The 2D data sets were subjected to Fourier and wavelet analyses. We obtained energy fluxes carried by waves with frequencies above the acoustic cutoff frequency $(\sim 5.2 \mathrm{mHz})$ at a height of $\sim 250 \mathrm{~km}$ in the solar atmosphere. We summarise the results as follows:

1. Both Fourier and wavelet analysis give total acoustic fluxes $F_{\text {ac,tot }}$ of $2600-3600 \mathrm{~W} \mathrm{~m}^{-2}$ at $250 \mathrm{~km}$ in the solar atmosphere. $65-75 \%$ of this flux is carried by waves in the frequency range $5.2-10 \mathrm{mHz}$, and $25-35 \%$ by waves at higher frequencies.

2. The waves appear in small-scale areas, of $1^{\prime \prime}-2$ " size for waves in the band of longer periods and at scales definitely below $1^{\prime \prime}$ in the short-period band. In the whole analysed period range from $50 \mathrm{~s}$ to $190 \mathrm{~s}$, the power occurs predominantly above inter-granular areas.

3. We do not see a large difference between the fluxes in pure inter-network areas and those in network.

The fluxes found here are higher by factors 3-6 than those obtained from filtergram sequences taken in space (Fossum \& Carlsson 2006, from TRACE; and Carlsson et al. 2007, from
HINODE). The fluxes found here are also higher by a factor of 2 than the value measured from acoustic waves at the same atmospheric height of $250 \mathrm{~km}$ by Straus et al. (2008). Radiative damping on the way to the onset of the chromospheric temperature rise in standard model atmospheres (Vernazza et al. 1981) could reduce the flux there to $1800-2500 \mathrm{~W} \mathrm{~m}^{-2}$ at $500 \mathrm{~km}$.

The finding that much of the flux is carried in the frequency band $5.2-10 \mathrm{mHz}$, i.e. at periods shorter than the acoustic cutoff period at $190 \mathrm{~s}$ down to $100 \mathrm{~s}$, supports the conjecture by Reardon et al. (2008). These authors suggest that the chromospheric power spectra measured by them in Ca II 8542 is a result from shock dissipation of waves near the cutoff frequency. We speculate that the mechanical flux of $1800-2500 \mathrm{~W} \mathrm{~m}^{-2}$ carried by acoustic waves to the chromosphere can serve, together with the flux in atmospheric gravity waves of $5000 \mathrm{~W} \mathrm{~m}^{-2}$ found by Straus et al. (2008), to produce at least a "warm" chromosphere, on average. This would then be the amount of basal flux to balance the radiative losses from chromospheric parts with little magnetic field (Schrijver 1987).

Our finding of most acoustic energy flux in the $5.2-10 \mathrm{mHz}$ range seemingly supports the view that waves with periods $U<$ $50 \mathrm{~s}$ are unimportant (e.g. Stein et al. 2004). Yet we emphasise that, from the observational point of view, we find many uncertainties in the frequency or period distribution of acoustic flux in the photosphere. Our knowledge is insufficient due to limited angular resolution and limited resolution in atmospheric height. Thus, we consider the problem of chromospheric heating not yet solved satisfactorily. Its solution is important because the chromospheres of the Sun and of late-type stars are the layers of the onset of exchange of mass, momentum, and energy between the stellar interior and the heliosphere and the interstellar medium.

To answer the question on chromospheric energy supply one would like to perform an observational experiment with high spatial resolution, much better than 0.5 , with high cadence, of the order of $10 \mathrm{~s}$, with very good discrimination in height, and with low noise. Possibly, a combination of signals originating from various heights will allow us to narrow the height ranges of signal formation. A multi-line capability would be very valuable to follow the wave propagation in various atmospheric heights. The next generation of detectors and data acquisition systems will certainly lead to high-cadence measurements. To achieve high spatial resolution and obtain simultaneously low-noise data is a task for telescopes with large aperture, such as GREGOR, EST, and ATST, including high-order Adaptive Optics. At the same time, such observations and their interpretation need to be guided by numerical simulations, as in Stein et al. (2004), Wedemeyer-Böhm et al. (2007), Straus et al. (2008), and most recently for photospheric magnetic field dynamics in Bello González et al. (2009). High Reynolds numbers are much desirable as well as small grid sizes in $(x, y, z, t)$. The goal would be to model the process of wave generation from turbulence and to follow their propagation through the solar atmosphere.

Acknowledgements. N.B.G. acknowledges financial support by Deutsche Forschungsgemeinschaft through grants KN 152/29-3 and KN 152/31-1. M.F.S. was supported by the ERASMUS programme of the European Union. O.O. thanks for support by Deutsche Forschungsgemeinschaft through grant KN 152/29-3 and by the Institut für Astrophysik of the Georg-August-Universität Göttingen. The Vacuum Tower Telescope is operated by the Kiepenheuer-Institut für Sonnenphysik, Freiburg, at the Spanish Observatorio del Teide of the Instituto de Astrofísica de Canarias. Wavelet software was provided by C. Torrence and G. Compo, and is available at URL: http://paos.colorado.edu/ research/wavelets. 


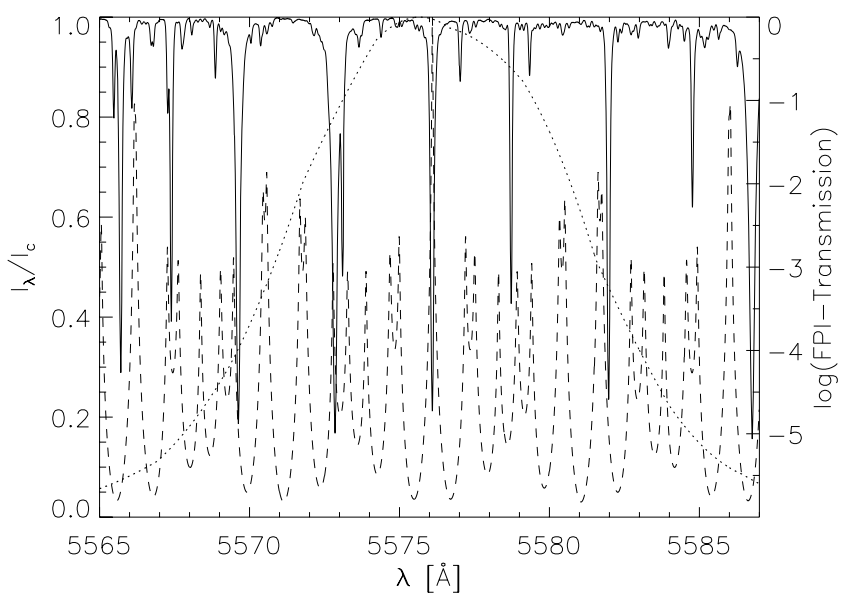

Fig. A.1. Solid: section of the solar spectrum from the Fourier Transform Spectrometer Atlas (FTS, Brault \& Neckel, quoted by Neckel 1999); dotted: transmission of the pre-filter with $\sim 10.7 \AA$ $F W H M$, from the data sheet of the manufacturer, normalised to its maximum; dashed, right ordinate: transmission of the Fabry-Perot spectrometer (FPI) normalised to maximum.

\section{Appendix A: Influence of pre-filter and noise filter}

We describe in this Appendix the influence of the rather broad pre-filter with $11 \AA F W H M$ and of the applied wavelength filter to smooth the line profiles. Figure A.1 depicts a $22 \AA$ wide section of the solar spectrum from the Fourier Transform Spectrometer Atlas (FTS, Brault \& Neckel, quoted by Neckel 1999). We adopt that the broadening of the spectral lines by the FTS is negligible, i.e. the FTS has an infinite spectral resolution. The transmission of the pre-filter, taken from the data sheet of the manufacturer, normalised to its maximum, is shown as the dotted curve. The solar spectrum multiplied with the transmission of the pre-filter gives the input to the Fabry-Perot interferometer (FPI). The transmission of the latter, also normalised to its maximum, is the dashed curve with the ordinate on the right side of Fig. A.1. We take finesses of $\mathcal{F}=40$ for both etalons with plate spacings of $1.101 \mathrm{~mm}$ and $1.408 \mathrm{~mm}$ and a neutral density filter between the etalons of 30\% absorption (Bello González \& Kneer 2008, Sect. 2.1 there).

One sees in the section of the solar spectrum several moderately strong to strong spectral lines whose positions are close to the transmission peaks of the FPI and whose intensities are not fully suppressed by the pre-filter. While scanning, i.e. moving the FPI transmission across the input by $\approx \pm 0.25 \AA$, these lines can have an influence on the velocity measurements of the Fe I $5576 \AA$ A line: an unidentified line (according to Moore et al. 1966) at $5566.1 \AA$ at a $1 \%$ level relative to the $5576 \AA$ line (FPI transmission multiplied with the transmission of the prefilter), the Fe I 5582.0 $\AA$ line at a $0.5 \%$ level, and the four Fe I lines at $5576.4 \AA$ A, $5569.6 \AA$, $5572.9 \AA$ A, $5573.1 \AA$ plus the $\mathrm{Ni}$ I line at $5578.7 \AA$. The influence of these latter five lines sums up to a level of $0.5 \%$ relative to the $5576 \AA$ line. The total amount of false line shifts from the above lines is thus at the $2 \%$ level. Tests were performed by shifting the whole solar spectrum, except the central part around $5576 \AA$, corresponding to a velocity of $1 \mathrm{~km} \mathrm{~s}^{-1}$. The resulting, false line shift in 5576 corresponded to $26 \mathrm{~m} \mathrm{~s}^{-1}$. We thus may neglect the shifts induced by the broad pre-filter.

The influence of the broad pre-filter on the profile of Fe I $5576 \AA$ is shown in Fig. A.2. There, the dashed profile is

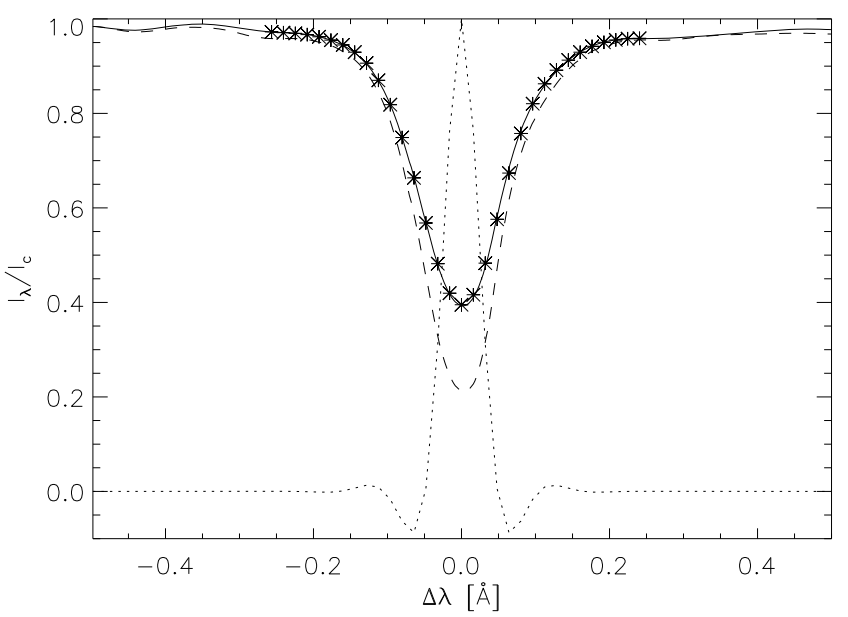

Fig. A.2. Dashed: section of spectrum from FTS around Fe I 5576 line; solid: same section after convolution with FPI transmission; dotted: line spread function of $49 \mathrm{~m} \AA F W H M$ for smoothing in wavelength; asterisks: line profile with observational wavelength spacing after smoothing in wavelength.

from the FTS atlas and the solid profile the FTS profile multiplied with the transmission of the pre-filter, then convolved with the FPI transmission and finally normalised to maximum intensity along the spectrum. One sees that the residual intensity of the convolved profile has increased by approximately a factor of two, as found above (Fig. 2 and Eq. (1) in Sect. 3.1).

For a precise measurement of the Doppler shifts we reduced the noise and the fluctuations of spectrometer transmission on short wavelength scales (cf. Bello González \& Kneer 2008, Sect. 2.1). For this we applied a filter whose Fourier transform is shown as the line spread function (LSP) by the dotted line in Fig. A.2. The LSP has an FWHM of $49 \mathrm{~m} \AA$. It preserves well the general shape of the line profile. Thus, the finally measured line intensities, indicated by the asterisks in Fig. A.2 do almost not differ from the original (though convolved with the FPI transmission) profiles. But the filter mixes intensities from the line wings to the line centre and vice versa. The result is a lowering of the formation height of the velocity fluctuations and a broadening (in height) of the velocity response function.

\section{References}

Anderson, L. S., \& Athay, R. G. 1989, ApJ, 346, 1010 Bello González, N., \& Kneer, F. 2008, A\&A, 480, 265

Bello González, N., Okunev, O. V., Domínguez Cerdeña, I., Kneer, F., \& Puschmann, K. G. 2005, A\&A, 434, 317

Bello González, N., Kneer, F., \& Puschmann, K. G. 2007, in Modern Solar Facilities - Advanced Solar Science, ed. F. Kneer, K. G. Puschmann, \& A. D. Wittmann, Universitätsverlag Göttingen, 217

Bello González, N., Yelles Chaouche, L., Okunev, O., \& Kneer, F. 2009, A\&A, 494, 1091

Bendlin, C., \& Volkmer, R. 1995, A\&AS, 112, 371

Bendlin, C., Volkmer, R., \& Kneer, F. 1992, A\&A, 257, 817

Biermann, L. 1948, ZAp, 25, 161

Carlsson, M., \& Stein, R. F. 1995, ApJ, 440, L29

Carlsson, M., \& Stein, R. F. 1997, ApJ, 481, 500

Carlsson, M., Judge, P. G., \& Wilhelm, K. 1997, ApJ, 486, L63

Carlsson, M., Hansteen, V. H., De Pontieu, B., et al. 2007, PASJ, 59, 663

Cuntz, M., Rammacher, W., \& Musielak, Z. E. 2007, ApJ, 657, L57

Cram, L. E., Keil, S. L., \& Ulmschneider, P. 1979, ApJ, 234, 768

de Boer, C. R. 1996, A\&AS, 120, 195

Durrant, C. J. 1979, A\&A, 73, 137

Eibe, M. T., Mein, P., Roudier, Th., \& Faurobert, M. 2001, A\&A, 371, 1128

Fawzy, D., Rammacher, W., Ulmschneider, P., Musielak, Z. E., \& Stępień, K. 2002, A\&A, 386, 971 
Fossum, A., \& Carlsson, M. 2005a, ApJ, 625, 556

Fossum, A., \& Carlsson, M. 2005b, Nature, 435, 919

Fossum, A., \& Carlsson, M. 2006, ApJ, 646, 579

Handy, B. N. Acton, L. W., Krankelborg, C. C., et al. 1999, Sol. Phys., 187, 229

Hasan, S. S., \& van Ballegooijen, A. A. 2008, ApJ, 680, 1542

Hasan, S. S., van Ballegooijen, A. A., Kalkofen, W., \& Steiner, O. 2005, ApJ, 631,1270

Jefferies, S. M., McIntosh, S. W., Armstrong, J. D., et al. 2006, ApJ, 648, L151

Kalkofen, W., Ulmschneider, P., \& Avrett, E. H. 1999, ApJ, 521, L141

Keller, C. U., \& von der Lühe, O. 1992, A\&A, 261, 321

Krieg, J., Wunnenberg, M., Kneer, F., Koschinsky, M., \& Ritter, C. 1999, A\&A, 343, 983

Krijger, J. M., Rutten, R. J., Lites, B. W., et al. 2001, A\&A, 379, 1052

Lighthill, M. J. 1952, Proc. Roy. Soc. London, A211, 564

Maltby, P., Avrett, E. H., Carlsson, M., et al. 1986, ApJ, 306, 284

Mein, N., \& Mein, P. 1980, A\&A, 84, 96

Mein, P. 1971, Sol. Phys., 20, 3

Moore, C. E., Minnaert, M. G. J., \& Houtgast, J. 1966, The Solar Spectrum $2935 \AA$ to $8770 \AA$, NBS, Monograph, 61

Musielak, Z. E., \& Ulmschneider, P. 2003a, A\&A, 400, 1057

Musielak, Z. E., \& Ulmschneider, P. 2003b, A\&A, 406, 725

Neckel, H. 1999, Sol. Phys., 184, 421

Puschmann, K. G., Kneer, F., Seelemann, T., \& Wittmann, A. D. 2006, A\&A, 445,337

Reardon, K. P., \& Cavallini, F. 2008, A\&A, 481, 897

Reardon, K. P., Lepreti, F., Carbone, V., \& Vecchio, A. 2008, ApJ, 683, L207

Schrijver, C. J. 1987, A\&A, 172, 111
Schwarzschild, M. 1948, ApJ, 107, 1

Shchukina, N., \& Trujillo Bueno, J. 2001, ApJ, 550, 970

Stein, R. F. 1967, Sol. Phys., 2, 385

Stein, R. F., Bogdan, T. J., Carlsson, M., et al. 2004, in SOHO 13 - Waves, Oscillations and Small-Scale Transient Events in the Solar Atmosphere:

A Joint View from SOHO and TRACE, ed. H. Lacoste, ESA SP-457, 93

Straus, T., Fleck, B., Jefferies, S. M., et al. 2008, ApJ, 681, L125

Torrence, C., \& Compo, G. P. 1998, Bull. Amer. Meteor. Soc., 79, 61

Tsuneta, S., Ichimoto, K., Katsukawa, Y., et al. 2008, Sol. Phys., 249, 167

Ulmschneider, P., Rammacher, W., Musielak, Z. E., \& Kalkofen, W. 2005, ApJ, 631, L155

Vecchio, A., Cauzzi, G., Reardon, K. P., Janssen, K., \& Rimmele, T. 2007, A\&A, 461, L1

Vecchio, A., Cauzzi, G., \& Reardon, K. P. 2009, A\&A, 494, 269

Vernazza, J. E., Avrett, E. H., \& Loeser, R. 1981, ApJS, 45, 635

Volkmer, R., Kneer, F., \& Bendlin, C. 1995, A\&A, 304, L1

von der Lühe, O. 1984, J. Opt. Soc. Am. A1, 510

von der Lühe, O., Soltau, D., Berkefeld, T., \& Schelenz, T. 2003, SPIE, 4853, 187

Wedemeyer-Böhm, S., Steiner, O., Bruls, J., \& Rammacher, W. 2007, in The Physics of Chromospheric Plasmas, ed. I. Dorotovic, P. Heinzel, \& R. Rutten, ASP Conf. Ser., 368, 93

Weigelt, G. P. 1977, Opt. Comm., 21, 55

Wunnenberg, M., Kneer, F., \& Hirzberger, J. 2002, A\&A, 395, L1

Yi, Z., \& Molowny Horas, R. L. 1992, in Proc. from LEST Mini-Workshop, Software for Solar Image Processing, ed. Z. Yi, T. Darvann, \& R. Molowny Horas, Oslo, Institute of Theoretical Astrophysics, 69 